\title{
Variaciones en el estilo estructural relacionado con anisotropias de basamento en el Valle Medio del Magdalena
}

\author{
Giovanny Jimenez ${ }^{1,2, *}$, Oscar López², Leonardo Jaimes², Ricardo Mier Umaña²,3 \\ ${ }^{1}$ Escuela de Geología, Grupo de Investigación GIGBA, Universidad Industrial de Santander, Bucaramanga, Colombia \\ ${ }^{2}$ Escuela de Geología, Universidad Industrial de Santander, Bucaramanga, Colombia \\ ${ }^{3}$ Grupo de investigación GIGHC, Universidad Industrial de Santander, Bucaramanga, Colombia
}

\begin{abstract}
Resumen
Con base en una revisión cartográfica en la zona central-este de la Cuenca VMM, la colección de datos estructurales y la elaboración de 3 cortes estructurales, se definieron 3 dominios estructurales en el área de estudio. El dominio este, dominio central y dominio oeste. El dominio este se caracteriza por la presencia de fallas inversas de alto ángulo y vergencia este, que involucran rocas Jurásicas. Los dominios central y oeste se caracterizan por la presencia de fallas inversas vergencia al oeste que involucran de manera general rocas del Cretácico inferior y rocas del PaleógenoNeógeno. El corte regional muestra que las fallas del dominio este corresponden a estructuras con inversión tectónica evidenciadas por los cambios de espesor de la Formación Girón; estas estructuras se definen como un estilo estructural dominado por una deformación tipo thick-skinned. Las estructuras de los dominios central y oeste muestran fallas inversas con despegues y rampas en las unidades Cretácicas y Paleógenas dominadas por una deformación tipo thin-skinned. El modelo regional planteado muestra un estilo estructural divergente y difiere del modelo estructural reportado en estudios previos. En el presente trabajo se propone una zona transversal para explicar las variaciones en el estilo estructural del Valle Medio del Magdalena de sur a norte. (c) Acad. Colomb. Cienc. Ex. Fis. Nat. 2016.
\end{abstract}

Palabras clave: estilo estructural, Valle Medio del Magdalena, inversión tectónica, dominios estructurales.

Structural style variation related with basement anisotropies in the Middle Magdalena Valley

\begin{abstract}
Based on a cartographic review of the central- eastern zone of the MMV Basin, the collection of structural data and 3 structural cross sections, it was possible to define three structural domains in the study area: The eastern domain, central domain and western domain. The eastern domain is characterized by east vergent and high angle reverse faults, involving the Girón Formation. Central and western domains are characterized by west vergent reverse faults involving Lower Cretaceous to Paleogene and Neogene rocks. A regional cross section shows that faults in the eastern domain correspond to tectonic inversion structures evidenced by changes in thickness of the Girón Formation. These structures are defined as a structural style dominated by thick-skinned deformation type. Structures of the central and western domains are characterized by flat-ramp reverse faults in the Cretaceous and Paleogene units dominated by thin-skinned deformation type. The regional model proposed shows a divergent structural style and differs from the structural model reported in previous studies. In this paper we propose a transverse zone to explain variations in the structural style of the Middle Magdalena Valley from south to north. (C) Acad. Colomb. Cienc. Ex. Fis. Nat. 2016.
\end{abstract}

Key words: Structural style, Middle Magdalena Valley, tectonic inversion, structural domains.

\section{Introducción}

El Valle Medio del Magdalena (VMM) es una cuenca sedimentaria que se caracteriza por registrar diferentes fases de deformación, que incluye eventos de extensión, compresión y transcurrencia (Cooper, et al., 1995; Taboada, et al., 2000; Sarmiento-Rojas, et al., 2006). Estudios previos en el sector Central-Sur de la Cuenca del VMM muestran un estilo estructural predominante con vergencia al Oeste y proponen una zona transversal hacia el norte (RestrepoPace, et al., 2004). Durante etapas de deformación en ambientes distensivos es posible originar anisotropías del basamento, las cuales en fases de deformación posteriores controlan y afectan el estilo estructural (Jiménez, et al, 2012). Teniendo en cuenta la geología de superficie es posible reconocer terminaciones abruptas de fallas, cambios en el rumbo de fallas y pliegues o cambios en la vergencia de la estructuras (Thomas, 1990; Thomas y Bayona, 2002; Jiménez, et al, 2012). En otros sistemas orogénicos, como

\footnotetext{
*Correspondencia:

Giovanny Jimenez, gjimenezd@gmail.com

Recibido: 15 de octubre de 2015

Aceptado: 1 de junio de 2016
} 
el cinturón sur de los Apalaches (Thomas, 1990; Thomas y Bayona, 2002), la terminación abrupta de pliegues y fallas o el cambio de vergencia de estructuras han sido relacionados con la presencia de estructuras transversales del basamento que controlan la deformación de la cobertera sedimentaria y causan variaciones en el estilo estructural. Las alineaciones de las terminaciones o de los cambios de vergencia tienen una dirección oblicua a perpendicular respecto al rumbo preferencial de las estructuras regionales. Estas alineaciones se agrupan en un área denominada zona transversal (Jiménez, et al, 2012). Estas zonas transversales se asocian a zonas de transferencia de cuencas de rift, como las definidas en el Rift de Suez y del Mar Rojo (Moustafa, 2002). El propósito principal de este artículo es realizar un control cartográfico, proponer un estilo estructural y evaluar la hipótesis de presencia de zonas transversales en el sector central - oriental del Valle Medio del Magdalena (Figura 1), como lo sugieren estudios previos (RestrepoPace, et al., 2004).

El estudio de zonas transversales es fundamental debido a que son un control fundamental en la cinemática de estructuras recientes y definen el estilo estructural regional de una cuenca. Para el caso del VMM estas zonas trasversales podrían ser un factor determinante en la localización y diferenciación de bloques con alto y/o bajo potencial petrolífero.
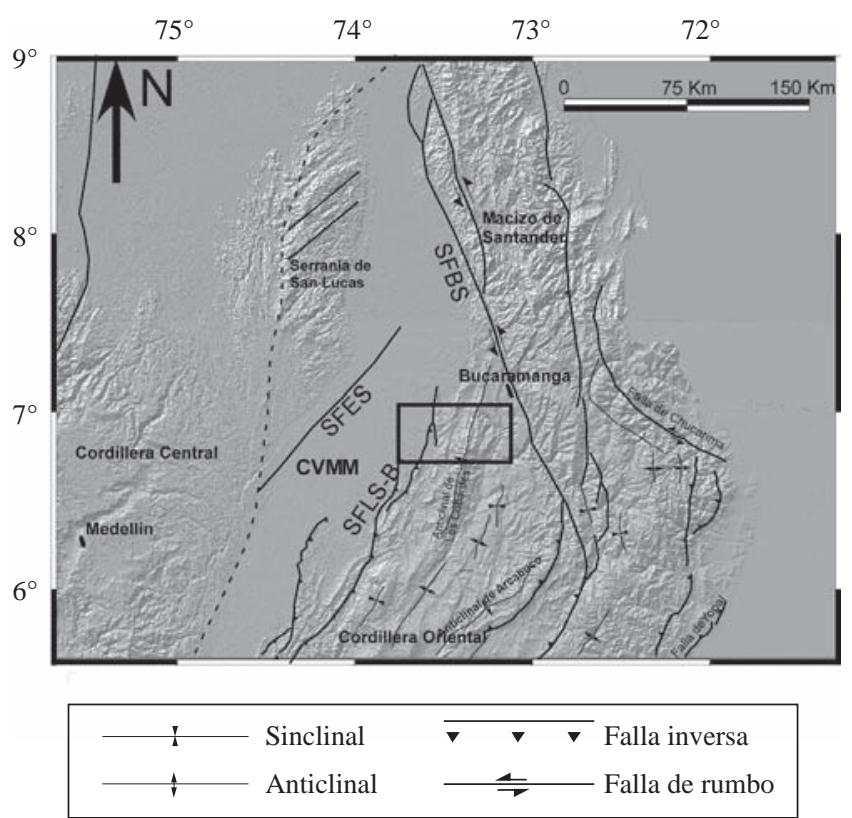

Figura 1. Modelo de Elevación Digital (DEM) donde se muestran los límites estructurales del Valle Medio del Magdalena y las cordilleras Oriental y Central. El área de estudio está representada por el recuadro CVMM = Cuenca Valle Medio del Magdalena, SFLS-B = Sistema de Fallas La Salina-Bituima, SFES= Sistema de Fallas Espíritu Santo, SFBS = Sistema de Fallas Bucaramanga Santa Marta.

\section{Marco geológico del Valle Medio del Magdalena}

Las rocas de los Andes del Norte en Colombia han experimentado diversas fases de deformación tectónica debidas a los movimientos e interacción entre las placas Nazca, Caribe y Suramericana (Cooper, et al., 1995; Taboada, et al., 2000; Sarmiento-Rojas, et al., 2006). La evolución tectónica de la Margen NW de Suramérica durante el Triásico-Jurásico Cretácico temprano, inicia con una fase de extensión con fallas normales que definen un hemigraben con el paleo-alto Macizo de Santander - Floresta el cual divide dos subcuencas: Tablazo-Magdalena al oeste y Cocuy al este (Cooper, 1995). Durante este periodo la extensión está dominada por una subsidencia tectónica (Fabre, 1987, Cooper, 1995), que permite la acumulación de los sedimentos continentales a marinos (Mojica y Franco, 1990). El Cretácico temprano es dominado por la subsidencia tectónica en una cuenca back arc (Fabre, 1987; Cooper, et al., 1995; Sarmiento-Rojas, et al., 2006). El Cretácico tardío es definido como un periodo dominado por una subsidencia termal y una subducción continúa al oeste (Cooper, et al., 1995; Sarmiento-Rojas, et al., 2006). La secuencia post rift o back arc en la Subcuenca Tablazo Magdalena está caracterizada por rocas marinas ricas en calizas, lodos orgánicos y areniscas (Cooper, et al., 1995; SarmientoRojas, et al., 2006). Las fases de compresión inician en el Maastrichtiano - Paleoceno temprano (Gomez, et al., 2003). Durante este periodo, la actual Cordillera Oriental se define como una cuenca segmentada y rellenada con depósitos synorogénicos y con pequeños bloques levantados (Bayona, et al., 2013; Parra, et al., 2012). Según Bayona, et al, (2013) En el Maastrichtiano a Paleoceno Medio la inclinación de la Cordillera Central favoreció la reactivación del borde oeste de la cuenca Cretácica. De acuerdo a Parra, et al, (2012) la exhumación del flanco oeste de la Cordillera Oriental inició en el Paleoceno.

Finalmente durante el Oligoceno Temprano - Mioceno se presenta la fase de inversión tectónica de la definiendo la configuración actual del VMM y la Cordillera Oriental (Colletta, et al., 1990; Cooper, et al., 1995; Mora, et al., 2010), (Figura 1).

\section{Geología estructural}

El VMM es definido como una depresión tectónica asimétrica y limitada por dos frentes cabalgantes de vergencia opuesta, (Córdoba, et al., 1996, Figura 1). Los límites estructurales del VMM los constituyen: al Norte, el sistema de fallas de Espíritu Santo (S.F.E.S); al Noreste, el sistema de fallas Bucaramanga-Santa Marta (S.F.B.S.M); al Sureste, el sistema de fallas Bituima y La Salina (S.F.B.S) y al Oeste, la Serranía de San Lucas y la Cordillera Central (Barrero, et al., 2007) (Figura 1).

El VMM está basculado hacia el este, afectado por pliegues y fallas (Pérez y Valencia, 1977, Mojica y Franco, 1990). La deformación incrementa hacia el Sureste definiendo un 
frente de deformación netamente compresional (Taborda, 1965). El límite Oeste del VMM puede ser definido como un límite con poca deformación suprayaciendo la Cordillera Central. La geometría de este límite se caracteriza por acuñamientos de las formaciones Cretácicas y Cenozoicas que buzan hacia el este (Mojica y Franco, 1990). Según Córdoba, et al., (2001) el VMM es definido estructuralmente en tres sectores. El sector oriental, el cual se caracteriza por estructuras con dirección NE-SW, que predominantemente corresponden a sistemas de cabalgamientos con vergencia Oeste. El sector central se encuentra poco deformado, donde se encuentran fallas normales fosilizadas. El sector occidental se caracteriza por la presencia de fallas inversas con componente transpresivo y vergencia Este.

\section{Estratigrafía}

Los primeros estudios estratigráficos que definen la nomenclatura estratigráfica del VMM, son reportados en Morales, et al., (1958) y modificados por Etayo-Serna y Laverde, (1985). De manera general la estratigrafía del VMM consiste de un basamento ígneo metamórfico de edades desde el Precámbrico al Paleozoico Inferior. Este basamento se encuentra suprayacido por una secuencia de rocas sedimentarias que van desde el Jurásico Tardío hasta el reciente (Schamel, 1991, Moreno, et al., 2011, Sarmiento L.F Rolón, 2004). Las principales características de la secuencia sedimentaria se pueden resumir como:

Rocas de edad Jurásico- Cretácico Inferior (Berriasiano): Corresponden a rocas volcano-sedimentarios en un sistema fluvial estructuralmente controlado (formaciones Girón, Arcabuco-Los Santos) (Etayo-Serna, 1968; Fabre, 1983). Estas unidades están siendo suprayacidas por depósitos de rocas sedimentarias siliciclásticas y calcáreas del Cretácico temprano en una plataforma marina somera, que fue más amplia que el actual VMM (Grupo basal conformado por las formaciones Cumbre, Rosablanca, Paja y Tablazo). Rocas de edad Albiano a Maastrichtiano: Corresponden a rocas de ambientes marinos someras acumuladas en una plataforma afectada por los cambios del nivel del mar (formaciones Simití y La Luna, (Rolón, 2004,). Rocas de edad Cretácico Superior a Paleoceno: Corresponden a rocas siliciclásticas marinas a paludales (formaciones Umir y Lisama) depositadas en ciclos regresivos-transgresivos (Rolón, 2004). Rocas de edad Paleógeno- Neógeno: Corresponden a rocas continentales depositadas en un ambiente fluvial y rocas clásticas lacustres (formaciones La Paz, Esmeraldas, Mugrosa, Colorado y Real) (Caballero, et al., 2010; Rolón, 2004).

\section{Metodologia}

La metodología usada en el presente trabajo consistió en la revisión cartográfica del sector $\mathrm{SW}$ del cuadrángulo H12 (Ward, et al., 1977a, 77b), donde se colectaron datos estratigráficos y estructurales con los cuales se generó el mapa regional (Figura 2). Se identificaron características litológicas y se controlaron los contactos litológicos de las formaciones Mesozoicas y Cenozoicas aflorantes. Se realizó una colección de datos estructurales (Rumbos y buzamientos de planos de estratificación, diaclasas, estrías y zonas de falla). Los datos estructurales se sometieron a la corrección por declinación magnética de $8^{\circ}$ Oeste (ocho grados), según los datos obtenidos de National Oceanic and Atmospheric Administration (NOAA). Se generó un mapa regional integrando los datos a la información disponible de mapas regionales (Ward, et al., 1973; Ward, et al., 1977a, 1977b; Royero y Clavijo, 2001; Beltrán y Quintero, 2008; Zafra, 2013). Finalmente se realizaron tres (3) cortes estructurales

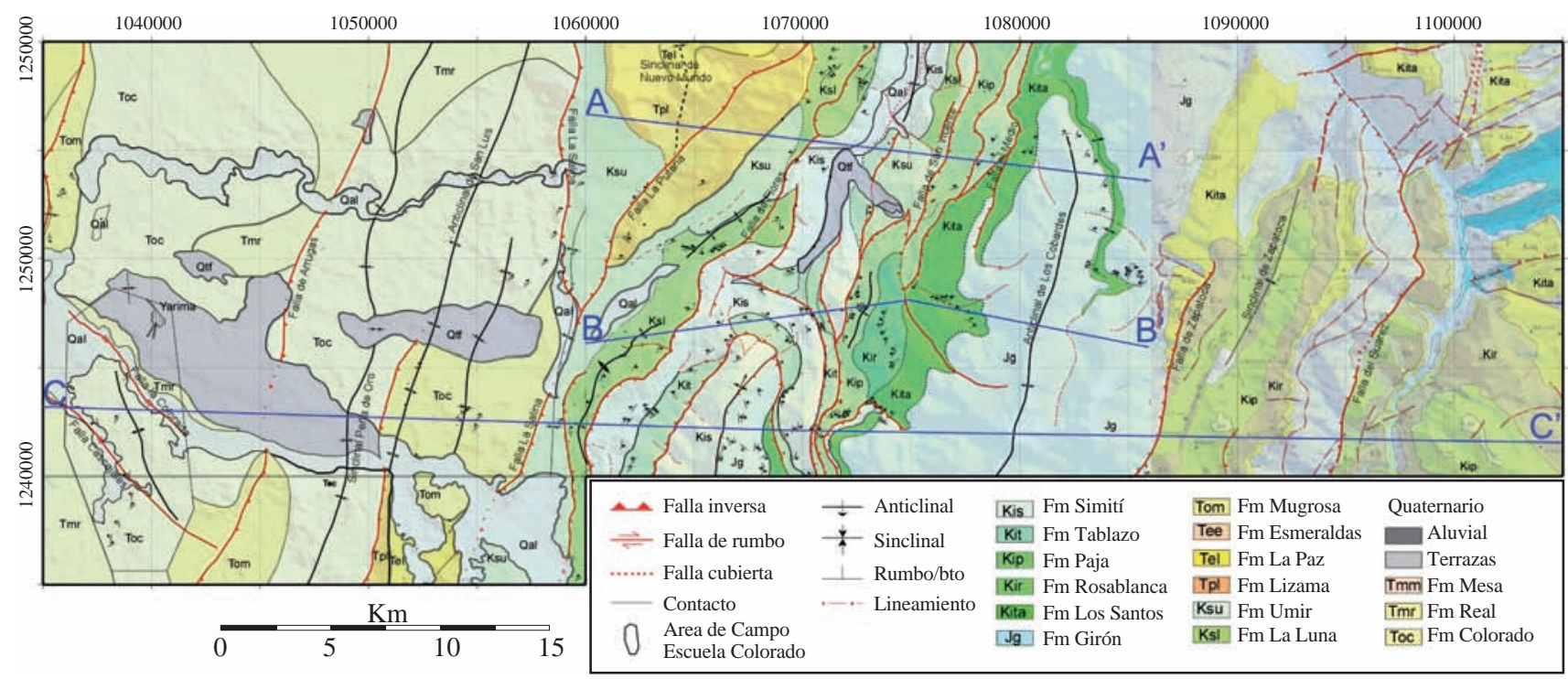

Figura 2. Mapa geológico del área de estudio donde se resaltan las estructuras geológicas principales y la localización de las líneas de cortes estructurales. Tomado y modificado de López y Jaimes, (2015); Ward, et al., (1977a, 1977b). 
del área de estudio: dos cortes representan la estructuración local y el otro corresponde a un marco regional del área de estudio. Los tres cortes fueron elaborados con una orientación aproximada E-O perpendicular al rumbo de las capas y estructuras. La construcción de los cortes se llevó a cabo siguiendo principios geométricos (Mitra, 1992; Suppe, 1983).

\section{Resultados}

\section{Cartografia}

Al integrar la revisión cartográfica realizada y la información geológica disponible se reconocen unidades estratigráficas de edades del Jurásico a Cretácico Superior y Neógeno (Figura 2). En la zona se reconocen estructuras plegadas y fallas con orientación principal NNE-SSW (Figuras 2, 3, 4 y 5). Las características estratigráficas y estructurales permiten definir tres dominios estructurales: Dominio este, dominio central y dominio oeste. El dominio este, se caracteriza estratigráficamente por la exposición de rocas Jurásicas (Formación Girón) y del Cretácico Inferior (Formaciones Los Santos, Rosa Blanca, Paja y Tablazo). Estructuralmente este dominio está definido por dos fallas inversas con vergencia al Este que corresponden a la Falla del Suarez y Zapatoca y con los pliegues Anticlinal de Los Cobardes y Sinclinal de Zapatoca. El bloque yacente de la Falla del Suarez corresponde a la Mesa de los Santos dominada por buzamientos bajos $\left(<30^{\circ}\right)$, donde afloran las formaciones Girón, Los Santos, Rosa Blanca, Paja y Tablazo (Figura 2).

El dominio central se caracteriza estratigráficamente por la exposición local de rocas del Jurásico (Formación Girón) y del Cretácico Inferior a Superior (Formación Tablazo, Simití, La Luna y Umir predominantemente). Estructuralmente el dominio central se localiza entre las Fallas San Vicente y Las Flores y se caracteriza por fallas inversas con vergencia Oeste. Los estratos en el bloque colgante de la Falla La Colorada buzan al este y en el bloque yacente buzan al oeste. Localmente en el dominio central se encuentran fallas con vergencia este (Figura 2).

El dominio oeste se caracteriza estratigráficamente por la pobre exposición de rocas, las cuales corresponden a rocas del Neógeno (Formaciones Colorado, Real y Mesa) así como depósitos aluviales recientes. Estructuralmente en este dominio se reconocen pliegues amplios y localmente se reconocen las fallas Arrugas, Cascajales y La Colorada (Figura 2).

\section{Cortes estructurales}

\section{Cortes geológicos locales.}

Los cortes geológicos locales A-A' y B-B' (Figura 3 y 4) se localizan en el dominio central. En el corte A-A' (Figura 3) se evidencia la vergencia Oeste de las fallas El Medio, San Vicente y Flores, las cuales tienen un despegue común y que sugiere una conexión con la Falla La Salina a profundidad. El Anticlinal de Los Cobardes y el Sinclinal de Nuevo Mundo en sus flancos oeste se encuentran afectados por dos fallas con vergencia al este. La falla que afecta al Anticlinal de Los Cobardes corresponde a un back thrust de las fallas El Medio. La falla que afecta al Sinclinal de Nuevo Mundo corresponde a la Falla La Putana que sería un back thrust de la Falla La Salina.

El corte B-B' (Figura 4) muestra una estructuración más sencilla con fallas con vergencia al oeste que corresponden a las fallas San Vicente y Flores con una falla menor localizada entre las dos anteriores y que localmente evidencia un back thrust asociado, afectando las unidades del Cretácico Inferior. Estas estructuras presentan un despegue común a profundidad y se evidencia una conexión con el frente de deformación de la Falla La Salina.

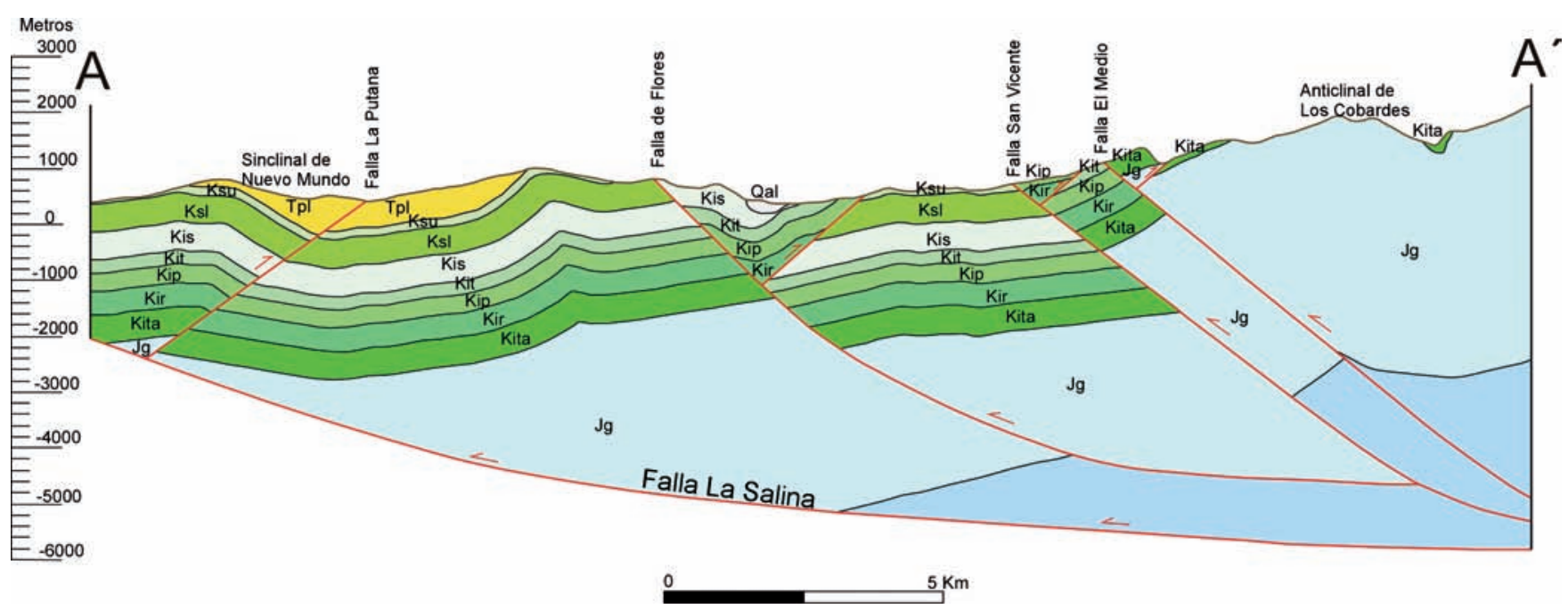

Figura 3. Corte estructural A-A’ donde se muestra la estructuración local del dominio central del área de estudio. Las escalas vertical y horizontal son iguales. Leyenda y localización del corte: ver Figura 4. Tomado y modificado de López y Jaimes, (2015) 


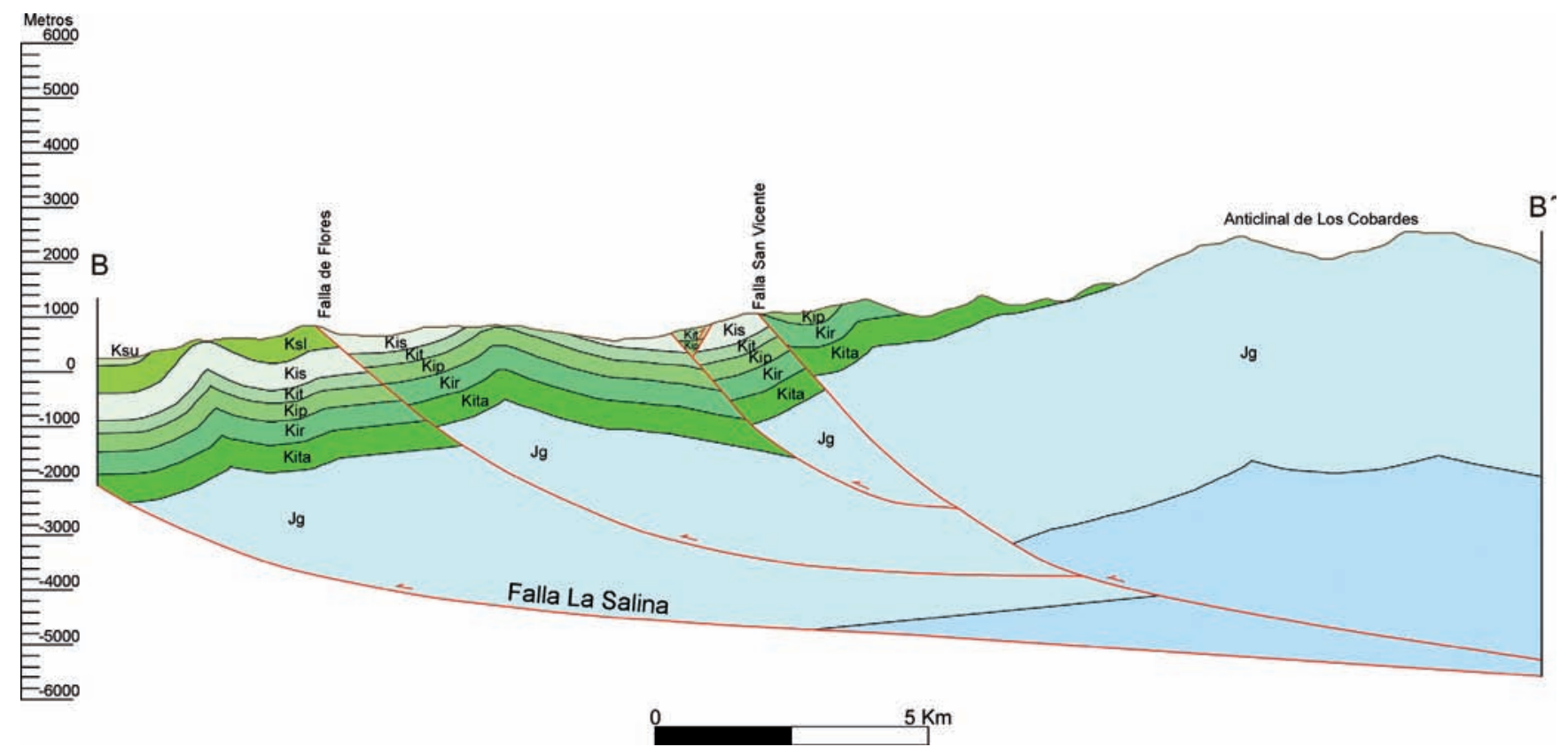

Figura 4. Corte estructural B-B' donde se muestra la estructuración local del dominio central del área de estudio. Las escalas vertical y horizontal son iguales. Leyenda y localización del corte: ver Figura 4. Tomado y modificado de López y Jaimes, (2015)

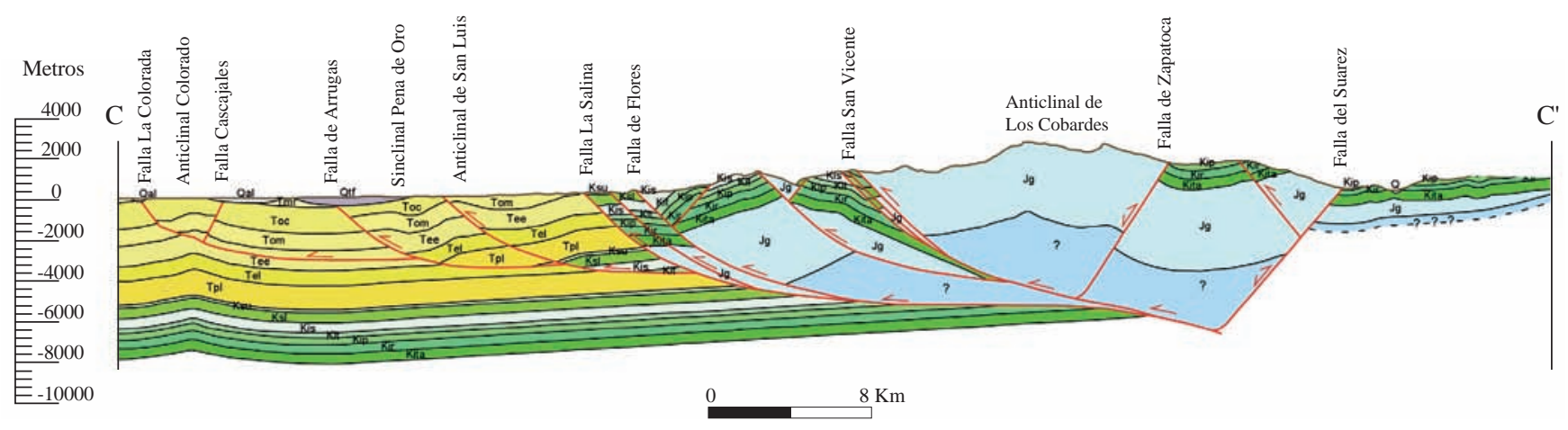

Figura 5. Corte estructural regional C-C' donde muestra la estructuración regional. Se incluyen los tres dominios, este, central y oeste del área de estudio. Las escalas vertical y horizontal son iguales. Leyenda y localización: ver Figura 4. Tomado y modificado de López y Jaimes, (2015)

\section{Corte estructural regional.}

El corte regional C-C' (Figura 5) corresponde a la integración de los tres dominios estructurales definidos en la cartografía. En el dominio este se resaltan las fallas del Suarez y Zapatoca, las cuales son fallas de alto ángulo y en el corte se marcan como fallas con inversión tectónica, debido a los cambios de espesor entre su bloque colgante y yacente. El dominio central se caracteriza por un despegue común en profundidad y otro más superior localizado en rocas del Cretácico Inferior (Formación Paja). El dominio estructural oeste en el corte C-C', se caracteriza por fallas con vergencia al oeste más espaciadas y causa pliegues más amplios. Los despegues en este dominio se localizan en el Cretácico Superior y Paleógeno.

\section{Discusión y conclusiones.}

De acuerdo a las características estratigráficas y estructurales se evidencia que el estilo estructural que define el sector este del VMM corresponde a un estilo de deformación tipo thick-skinned y que varía hacia sector central del VMM a un estilo de deformación tipo thin-skinned (Figuras 2, 3, 4 y 5). Los cortes estructurales evidencian que las fallas con vergencia este, corresponden back thrust asociados a fallas principales (Figura 5). Estos back thrust se encuentran tanto en los dominios de formación tipo thin-skinned y thick-skinned (Figuras 3, 4 y 5).

En el dominio este del área de trabajo se evidencia que las rocas Jurásicas (Formación Girón), se involucran en la dinámica de acortamiento (Figura 2 y 5). Los cambios de 


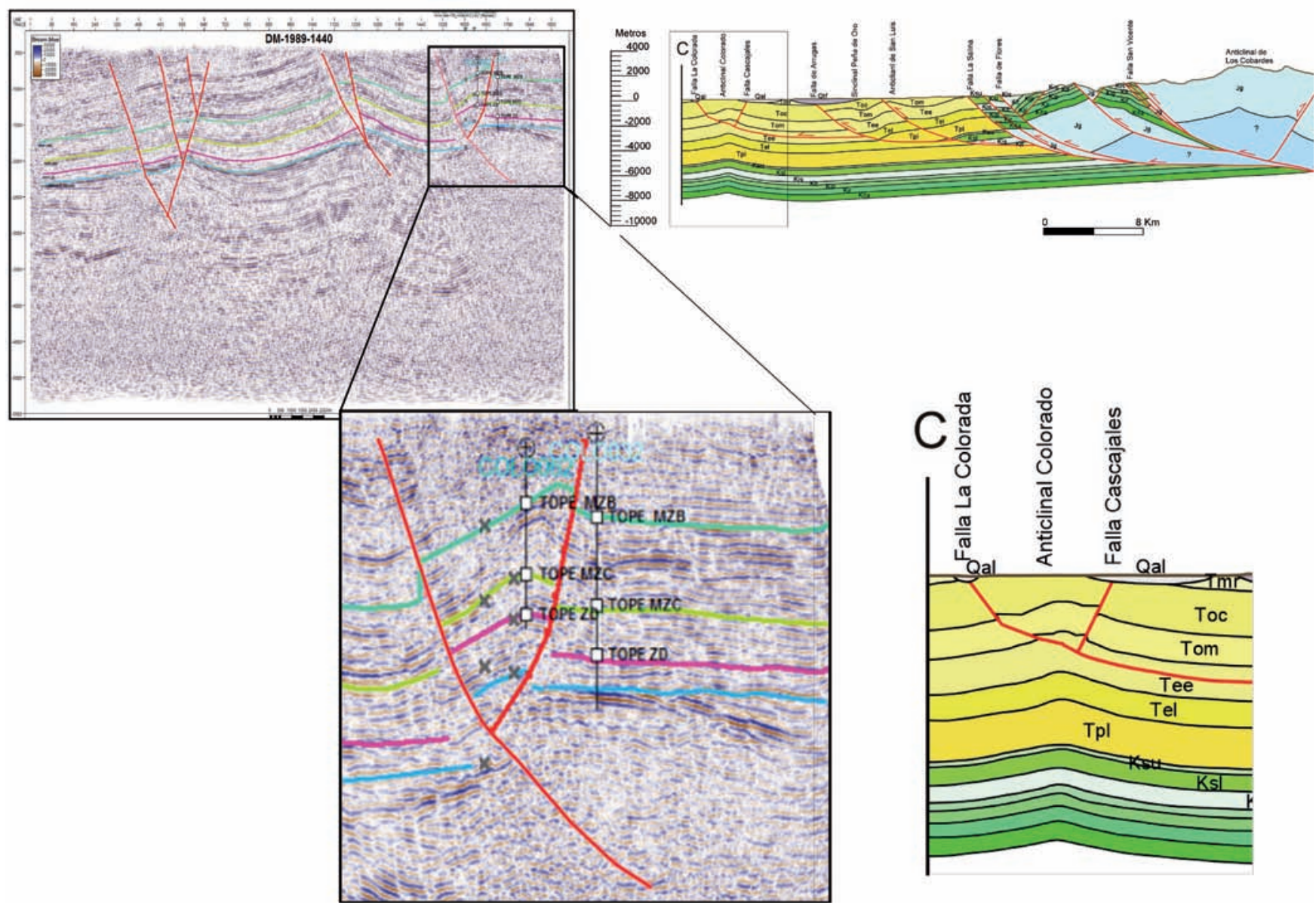

Figura 6. Modelo estructural del Campo Escuela Colorado. Estructura anticlinal definida por la línea sísmica DM-1989-1440 y el modelo obtenido del Corte C-C' en Campo Colorado. Tomado y modificado de Chajín, et al., (2013); López y Jaimes, (2015).

espesor registrados en la Formación Girón e incluso en la Formación Simití podrían relacionarse a la presencia de fallas normales. Los cambios de espesor definidos en los bloques, colgante y yacente de las fallas Suarez y Zapatoca permiten inferir que estas estructuras corresponden a estructuras de inversión tectónica (Figura 2 y 5).

Hacia el dominio central localmente se reconocen afloramientos de la Formación Girón, mientras que en el dominio oeste está ausente. Esto implica que los desplazamientos verticales de las fallas han de ser mucho menores y se encuentran despegues exclusivamente en rocas del Cretácico y Cenozoico (Figuras 3, 4 y 5).

Chajín, et al., (2013) con base en información de subsuelo proponen para el Campo Escuela Colorado un anticlinal asimétrico, con un eje N-S y cabeceo al norte (Figura 6). El anticlinal está asociado a la Falla Colorado, la cual presenta un back thrust. Por otro lado, con información de superficie estas estructuras se definen como fallas normales con componente sinestral (Zafra, 2013). El modelo presentado en el corte C-C' (Figura 6) es coherente con la geometría presentada por Chajín, et al., (2013).
En general se reconoce una diferencia notable en el estilo estructural del sector este del VMM respecto al sector central y sur del VMM. El sector este del VMM se caracteriza por fallas de alto ángulo, con vergencia al este y que involucran rocas Jurásicas, con cambios de espesor en las unidades. Mientras que el sector central se caracterizan por la presencia de cabalgamientos y pliegues asociados con vergencia oeste similar al estilo estructural definido por (Restrepo-pace, et al., 2004). El estilo estructural definido para el sector sur del VMM involucra un sistema de cabalgamientos con vergencia al oeste con una zona de transferencia hacia el norte (Restrepo-Pace, et al., 2004). Con el fin de explicar los cambios en el estilo estructural de sur a norte se propone una zona de transferencia, como posible mecanismo responsable de la generación de estructuras con vergencia al este. Según Vauchez, et al, (1998) un modelo realista de la litosfera está representado por heterogeneidades mecánicas y anisotropías. De acuerdo a Soto, et al, (2003) el espesor de sedimentos pre tectónicos puede ser variable debido a 1) actividad de fallas normales, 2) presencia de altos de basamento 3) proximidad del área fuente y 4) compactación diferencial junto a cambios 
litológicos y reológicos. Los cambios laterales de espesor junto una compresión oblicua controlan el desarrollo de cabalgamientos curvos o segmentados (Soto, et al, 2003).

Según el modelo las anisotropías de basamento asociadas a fallas normales originadas durante el Triásico-Jurásico a Cretácico temprano, causan variaciones laterales de espesores, como los reportados en la Formación Girón (Figuras 3, 4, 5). En las etapas posteriores de compresión las antiguas fallas normales se caracterizan por sufrir inversión tectónica e involucran una deformación tipo thick-skinned con cambios en la vergencia de las fallas y pliegues asociados.

\section{Agradecimientos}

El presente trabajo hace parte del Proyecto Modelo Geológico de Campo Escuela Colorado, los autores agradecemos a Campo Escuela Colorado y a la Vicerrectoría de Investigación y Extensión (VIE) de la UIS por el apoyo económico, administrativo y social para llevar a cabo esta y otras investigaciones relacionadas con el proyecto. También agradecemos a la continua colaboración de la escuela de Geología.

\section{Conflicto de intereses}

Los autores declaran no tener conflicto de intereses.

\section{Bibliografía}

Barrero, D., Pardo, A., Vargas, C., Martínez, J. (2007). Colombian sedimentary basins: Nomenclature, Boundaries and Petroleum Geology, a New Proposal. Agencia Nacional de Hidrocarburos - ANH - Edited and compiled by: ANH and B\&M Exploration Ltda. Bogotá.

Bayona, G, Cardona. A, Jaramillo. C, Mora. A, Montes. C, Caballero. V, Mahecha. H, Lamus-Ochoa. F, Montenegro. O, Jimenez. G, Mesa. A, Valencia. A (2013), Onset of fault reactivation in the Eastern Cordillera of Colombia and proximal Llanos basin; response to Caribbean-South American collision in early Palaeogene time, in ThickSkin-Dominated Orogens: From Initial Inversion to Full Accretion, edited by M. Nemčok, A. R. Mora, and J. W. Cosgrove, Geol. Soc. London, Spec. Publ., 377, doi:10.1144/ SP377.5.

Beltrán, A., Quintero, C. (2008). Geología de la Plancha 119, Barrancabermeja. Escala 1: 100.000. Ingeominas. Bogotá.

Caballero, V., Parra, M., Mora, A. (2010). Levantamiento de la Cordillera Oriental de Colombia durante el Eoceno tardío - Oligoceno temprano: proveniencia sedimentaria en el sinclinal de Nuevo Mundo, cuenca Valle Medio del Magdalena. Boletín de Geología Vol. 32, N¹, Enero-Junio de 2010. p 45-77.

Chajín, P., Mier, R., Palmera, T. Delgado, L., Luna, J. (2013). Reinterpretación del Modelo Estructural del Campo Escuela Colorado, VMM, Colombia. En. XV Congreso Colombiano de Petrólero y Gas. Noviembre 20 al 22-Corferias. Bogotá.

Colletta, B., Hebrard, F., Letouzey, J., Werner, P., and Rudkiewicz. J. (1990). Tectonic style and crustal structure of the Eastern Cordillera (Colombia) from a balanced cross section, in Petroleum and Tectonics in Mobile Belts, edited by J. Letouzey, pp. 81-100, Technip, Paris.

Cooper, M. A., Addison, F. T., Álvarez, R., Coral, M., Graham, R. H., Hayward, S. H., Martínez, J., Naar, J., Peñas, R., Pulham, A. J., Taborda , A. (1995). Basin development and tectonic history of the Llanos Basin, Eastern Cordillera, and Middle Magdalena Valley, Colombia: American Association of Petroleum Geologists Bulletin, v. 79, no. 10, p. 1421-1443.

Córdoba, F., Rolón, L., Buchelli, F., Suarez, M. (1996). Provincia petrolífera del Valle Medio del Magdalena, Colombia. Empresa Colombiana de Petróleos (ECOPETROL). p. 603605. Santafé de Bogotá.

Córdoba, F., López, C., Rolón, L., Buchelli, F., Sotelo, C. (2001). Proyecto evaluación regional del Valle Medio del Magdalena y Cordillera, Ecopetrol, informe interno: Bogotá.

Dengo, C. A., Covey, M.C. (1993). Structure of the Eastern Cordillera of Colombia: implications for trap styles and regional tectonics. American Association of Petroleum Geologists Bulletin 77: 1315-1337.

Etayo-Serna, F. (1968). El sistema Cretáceo en la región de Villa de Leiva y zonas próximas: Geología Colombiana, v. 5, p. 5-74.

Etayo-Serna, F. y F. Laverde. (1985). Proyecto Cretácico. Publicaciones Especiales del INGEOMINAS, 16: 1-450. Bogotá.

Fabre, A. (1983). La subsidencia de la Cuenca del Cocuy (Cordillera Oriental de Colombia) durante el Cretáceo y el Terciario Inferior. Primera parte: Estudio cuantitativo de la subsidencia: Geología Norandina, v. 8, p. 22-27.

Gomez, E., T. Jordan, R. Almandiger, K. Hegarty, S. Kelly, and M. Heizler (2003), Controls on architecture of the Late Cretaceous to Cenozoic southern Middle Magdalena Valley Basin, Colombia, Geol. Soc. Am. Bull., 115: 131-147, doi: 10.1130/00167606(2003)115<0131:COAOTL> 2.0.CO;2.

Ghiglione, Suarez. F, Ambrosio. A, Da Poian. G, Cristallini. E.O, Pizzio M.F y R. M. Reinoso (2009). Structure and evolution of the austral basin fold-thrust belt, Southern Patagonian Andes. Rev. Asoc. Geol. Argent. Vol. 65, n.1, p. 215-226.

Jiménez, G., Rico, J., Bayona, G., Montes, C., Rosero, A., Sierra, D., 2012. Analysis of curved folds and fault/fold terminations in the souther Upper Magdalena Valley of Colombia. Journal of South American Earth Sciences, 39: 184-201.

López. O. A y Jaimes. E. L. (2015). Contribución al conocimiento geológico del sector Sureste de la Plancha 120-II-C, Valle Medio del Magdalena. Tesis de Grado, Universidad Industrial de Santander

Mitra, S. (1992). Balanced structural interpretations in fold and thrust belts. En Mitra, S. Y Fisher, G. Structural geology of fold and thrust belt, Baltimore, USA, Johns Hopkins University, p: 53-77. 
Mojica, J. y Franco, R. (1990): Estructura y Evoluci6n Tect6nica del Valle Medio y Superior del Magdalena.- Geología. Colombiana 17, pp.41-64, 14

Mora, A., M. Parra, M. R. Strecker, E. R. Sobel, G. Zeilinger, C. Jaramillo, S. Ferreira Da Silva, and M. Blanco (2010), The eastern foothills of the Eastern Cordillera of Colombia: An example of multiple factors controlling structural styles and active tectonics, Geol. Soc. Am. Bull., 122: 1846-1864, doi:10.1130/B30033.1.

Morales, L. G. (1958). General geology and oil occurrences of Middle Magdalena Valley, Colombia. In L. G. Weeks ed. A symposium conducted by A.A.P.G.: Habitat of Oil, p. 641-695.

Moreno, C. J., Horton, B. K., Caballero, V., Mora, A., M., Sierra, J. (2011). Depositional and provenance record of the Paleogene transition from foreland to hinterland basin evolution during Andean orogenesis, northern Middle Magdalena Valley Basin, Colombia. Journal of South American Earth Sciences 32: 246-263.

Moustafa, A., 2002. Controls on the geometry of transfer zones in the Suez rift and northwest Red Sea: Implications for the structural geometry of rift system. AAPG Bulletin, Vol. 86, No.6, pp. 979-1002.

Parra, M., A. Mora, C. López, L. E. Rojas, and B. K. Horton (2012), Detecting early shortening and deformation advance in thrust-belt hinterlands: Example from the Colombian Andes, Geology, 40: 175-178, doi:10.1130/ G32519.1.

Pérez, G., Valencia, M. (1977). Evaluación geológica del Valle Medio del Magdalena.- Informe 588, Div. Explor. Depto. Geol., Ecopetrol, Bogotá.

Restrepo - Pace, P. A., F. Colmenares, C. Higuera, and M. Mayorga, (2004). A Fold and thrust belt along the western flank of the Eastern Cordillera of Colombia-Style, kinematics, and timing constraints derived from seismic data and detailed surface mapping, in K. R. McClay, ed., Thrust tectonics and hydrocarbon systems: AAPG Memoir 82, p. 598-613.

Rolón, L., F. (2004). Structural Geometry of the Jura-Cretaceous Rift of the Middle Magdalena Valley Basin-Colombia. Department of Geology and Geography, Morgantown, WV.

Royero, J., Clavijo, J. (2001). Mapa Geológico Generalizado Departamento de Santander. Bogotá: Ingeominas.

Sarmiento-Rojas, L. F., J. D. Van Wess, and S. Cloetingh (2006), Mesozoic transtensional basin history of the Eastern Cordillera, Colombian Andes: Inferences from tectonic models, J. S. Am. Earth Sci., 21: 383-411, doi: 10.1016/j.jsames.2006.07.003.
Schamel, S., (1991). Middle and Upper Magdalena Basins, Colombia: Chapter 10. M 52: Active Margin Basins, p. 283-301.

Soto.R, Storti. F, Casas. A.M y C. Faccenna (2003). Influence of along-strike pre-orogenic sedimentary tapering on the internal architecture of experimental thrust wedges. Geological Magazine, 140 (3): 253-264.

Suppe J. (1983). Geometry and kinematic of fault-bend-folding. en Foster N.H. y Beaumont E.A. (eds.) Structural concepts and techniques I. Basic concepts, folding and structural techniques. AAPG publicación especial No 9. P. 422-461.

Taboada, A., Rivera, L. A., Fuenzalida, A., Cisternas, A., Philip, H., Bijwaard, H., Olaya, J., Rivera, C. (2000). Geodynamics of the northern Andes: subductions and intracontinental deformation (Colombia). Tectonics 19: 787-813

Taborda, B. (1965). The geology of the de Mares Concession in Geological Field Trips, Colombia. 1958 -1978: Colombian Society of Petroleum Geologists and Geophysicists, p. 119-159.

Thomas, W. A., 1990. Control of locations of transverse zones in Thrust belts. Eclogae Helvetiae 83: 727-744

Thomas. W., A., y Bayona, G., 2002. Palinspastic restoration of the Anniston transverse zone in the Appalachian Thrust Belt, Aabama. Journal of structural geology 24: 797-826.

Vauchez. A, Tommassi. A y G Barruol. (1998). Rheological heterogeneity, mechanical anisotropy and deformation of the continental lithosphere. Tectonophysics 296: 61-86.

Ward, D. E., Goldsmith R., Jimeno, A., Cruz, J., Restrepo, H., Gómez, E. (1973). Mapa Geológico de Colombia Cuadrángulo H-12, Bucaramanga. Panchas 109 Rionegro 120 Bucaramanga, Cuadrángulo H-13, Pamplona. Planchas 110 Pamplona - 121 Cerrito. Escala 1: 100.000. Memoria explicativa. Colombia: Colombia Ministerio de Minas y Petróleos, Inst. Nal. Inv. Geológico - Mineras.

Ward, D. E., Goldsmith, R., Jimeno, A., Cruz, J., Restrepo, H., Gómez, E. (1977a). Geología de la Plancha 120, Bucaramanga. Ingeominas. Bogotá.

Ward, D. E., Goldsmith, R., Jimeno, A., Cruz J., Restrepo, H., Gómez E. (1977b). Mapa Geológico del Cuadrángulo H-12, Bucaramanga. Escala 1: Ingeominas.

Zafra, E. (2013). Cartografía geológica del Campo Colorado a escala 1: 25.000. Universidad Industrial de Santander. Bucaramanga. 